Springer LNP 622: Irreversible Quantum Dynamics, F. Benatti, R. Floreanini Eds., p. 233-242 (2003)

\title{
The Quantum Jump Approach and Quantum Trajectories
}

\author{
Gerhard C. Hegerfeldt
}

Institut für Theoretische Physik, Universität Göttingen, Germany

\begin{abstract}
A both conceptually and practically useful tool for the description of modern experiments on a single atom or a single system is the quantum jump approach with the associated quantum trajectories. The underlying ideas are outlined and then extended to moving systems. A new application to the problem of quantum arrival times is indicated.
\end{abstract}

\section{Introduction}

Before the advent of atom and ion traps only experiments involving many atoms were possible, e.g. atoms in an atomic beam or in a gas. With a beam one would have a repetition of measurements on an ensemble, while experiments on atoms in a gas - dilute and with no cooperative effects - can often be viewed as a simultaneous measurement on an ensemble. This is well adapted to the statistical interpretation of quantum mechanics. With atom traps, however, and with laser cooling it became possible to store a single atom (ion) in a trap for hours or days and to do experiments with it, e.g. study its interaction with light, microwave radiation or with other atoms.

As a more interesting example of what can happen consider the macroscopic dark periods ('electron shelving') of a single three-level atom as proposed by Dehmelt [1]. The atom is supposed to have a ground state 1 and two excited states 2 and $2^{\prime}$ where the former is strongly coupled to 1 and decays rapidly, while the latter is metastable. The $1-2$ transition is strongly driven by a laser, and the $1-2^{\prime}$ transition is weakly driven.

Semiclassically the behavior of such a single atom is easy to understand. The electron makes rapid transitions between levels 1 and 2, accompanied by a stream of spontaneous photon emissions, in the order of $10^{8} \mathrm{~s}^{-1}$. These can be detected (and even seen by the eye). From time to time the weak driving of the $1-2^{\prime}$ transition manages to put the electron into the metastable level $2^{\prime}$ where it stays for some time ('shelving'). During this time the stream of spontaneous photons is interrupted and there is a dark period. Then the electron jumps back to level 1 and a new light period begins. In an ensemble of such atoms (e.g. gas with no cooperative effects) light and dark periods from different atoms will overlap, and consequently one will just see diminished fluorescence. Only light and dark periods from a single or a few atoms are directly observable.

In contrast to these semi-classical arguments, quantum mechanically the atom 
will always be in a superposition of the three state $|1\rangle,|2\rangle$, and $\left|2^{\prime}\right\rangle$ and never strictly in state $\left|2^{\prime}\right\rangle$ for an extended period, i.e. there will always be a small admixture of $|2\rangle$. Since $|2\rangle$ decays rapidly the question arises if the dark periods still occur. Experiments [2] and early theoretical treatment [3] have answered this affirmatively. The duration of the dark periods is random and can be seconds or even minutes.

To treat problems like these involving a single system Wilser and the author [4, $5,6]$ developed the quantum jump approach which is equivalent and simultaneous to the Monte-Carlo wave function approach (MCWF) [7] and to the quantum trajectory approach [8]. Our approach is based on standard quantum mechanics and nothing new to the latter is added or required. In the next section we will give a short exposition of the quantum jump approach with its associated random ('quantum') trajectories for a single radiating system at rest. This is then generalized to a moving system. As a new application a model for quantum arrival times is indicated.

\section{Repeated gedanken measurements on a single system: Jumps and random trajectories}

We briefly review the ideas underlying our approach to a system radiating photons. It is based on standard quantum mechanics and adds no new assumptions or properties to the latter. It has turned out to be a practical tool for questions concerning a single system and often has technical and conceptual advantages. More details as well as applications can be found in Refs. $[4,6,9,10,11,12]$ and in the surveys $[13,14]$.

It is intuitively reasonable that it should make no difference physically whether or not the photons radiated by an atom are detected and absorbed once they are sufficiently far away. It therefore suggests itself to employ gedanken photon measurements, over all space and with ideal detectors, at instances a time $\Delta t$ apart[15]. For a single driven atom this may look as in Fig. 1. Starting in some initial state

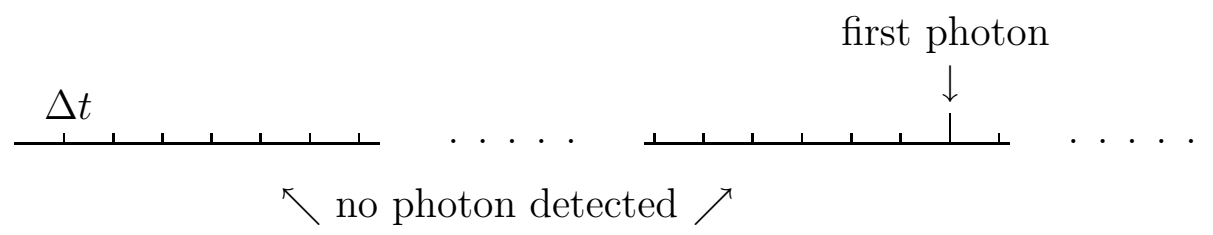

Figure 1: Repeated photon measurements.

with no photons (the laser field is considered as classical), at first one will detect no emitted photon in space and then at the $n_{1}$-th measurement a first photon will be detected (and absorbed), the next photon at the $n_{2}$-th measurement and so on.

Limits on $\Delta t$ : (i) Ideally one would like to let $\Delta t \rightarrow 0$ to simulate continuous measurements. But this is impossible in the framework of standard quantum mechanics with ideal measurements due to the quantum Zeno effect [16]. (ii) Intuitively, $\Delta t$ 
should be large enough to allow the photons to get away from the atom. (iii) $\Delta t$ should be short compared to level life-times. This leads to the requirement [17]

$$
\Delta t \cong 10^{-13}-10^{-10} \mathrm{~s} .
$$

We translate these gedanken measurements on a single atom into an ensemble description as follows. Let $\mathcal{E}$ be an ensemble of many atoms, each with its own quantized radiation field, of which our individual atom plus field is a member. At time $t_{0}=0$ the ensemble is described by the state $\left|0_{p h}\right\rangle\left|\psi_{A}\right\rangle$. Now we imagine that on each member of $\mathcal{E}$ photon measurements are performed at times $\Delta t, \ldots, n \Delta t, \ldots$. Now we denote, for $n=1,2, \ldots$, by $\mathcal{E}_{0}^{(n \Delta t)}$ the sub-ensemble which consists of all systems of $\mathcal{E}$ for which at the times $\Delta t, \ldots, n \Delta t$ no photon was detected. This is depicted in Fig. 2 where our individual system, atom plus radiation field, is denoted by a dot $\cdot$, and it is a member of $\mathcal{E}_{0}^{(n \Delta t)}$ for $n<n_{1}$.

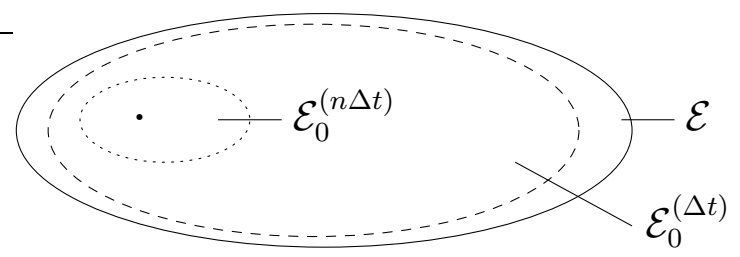

Figure 2: Ensemble $\mathcal{E}$ and sub-ensembles. The dot denotes our single system.

Now one can proceed by ordinary quantum mechanics and the von NeumannLüders reduction rule [18]. Let $\mathcal{P}_{0}$ be the projector onto the no-photon subspace,

$$
\mathcal{P}_{0} \equiv\left|0_{p h}\right\rangle \mathbf{1}_{A}\left\langle 0_{p h}\right|
$$

and let $U\left(t, t_{0}\right)$ be the complete time-development operator, including the laser driving and the interaction of the atom with the quantized radiation field. Then the sub-ensemble $\mathcal{E}_{0}^{(\Delta t)}$ is described by

$$
\mathcal{P}_{0} U(\Delta t, 0)\left|0_{p h}\right\rangle\left|\psi_{A}\right\rangle
$$

and the sub-ensemble $\mathcal{E}_{0}^{(n \Delta t)}$ by

$$
\mathcal{P}_{0} U(n \Delta t,(n-1) \Delta t) \mathcal{P}_{0} \ldots \mathcal{P}_{0} U(\Delta t, 0)\left|0_{p h}\right\rangle\left|\psi_{A}\right\rangle \equiv\left|0_{p h}\right\rangle\left|\psi_{A}(t)\right\rangle
$$

where we have put $t \equiv \Delta t$. The relative size of the sub-ensemble $\mathcal{E}_{0}^{(n \Delta t)}$ is the probability to find a member of $\mathcal{E}$ in $\mathcal{E}_{0}^{(n \Delta t)}$ and is given by the norm-square of the above expression. Hence

$$
P_{0}(t) \equiv \|\left|\psi_{A}(t)\right\rangle \|^{2}
$$

is the probability to find no photon until time $t=n \Delta t$. To calculate $\left|\psi_{A}(t)\right\rangle$ we note that

$$
\mathcal{P}_{0} U\left(t^{\prime}+\Delta t, t^{\prime}\right) \mathcal{P}_{0}=\left|0_{p h}\right\rangle\left\langle 0_{p h}\left|U\left(t^{\prime}+\Delta t, t^{\prime}\right)\right| 0_{p h}\right\rangle\left\langle 0_{p h}\right|
$$


and that the inner expression is a purely atomic operator which is easily obtained by second order perturbation theory. For $\Delta t$ in the above limits one then obtains, on a coarse-grained time scale (for which $\Delta t$ is very small), that the time development of $\left|\psi_{A}(t)\right\rangle$ is given by a 'conditional', or 'reduced', non-Hermitian Hamiltonian $H_{\text {cond }}$ in the atomic Hilbert space where, for an $N$-level atom,

$$
H_{\text {cond }}=H_{A}(t)-i \hbar \Gamma
$$

with $H_{A}(t)$ the atomic part of the Hamiltonian, including the laser driving, and

$$
\begin{aligned}
\Gamma & \equiv \sum_{\substack{\alpha i j \\
\alpha<i, j}} \Gamma_{i \alpha \alpha j}|i\rangle\langle j| \\
\Gamma_{i j l m} & \equiv e^{2}\langle i|\mathbf{X}| j\rangle \cdot\langle l|\mathbf{X}| m\rangle\left|\omega_{l m}\right|^{3} /\left(6 \pi \epsilon_{0} \hbar c^{3}\right)
\end{aligned}
$$

and $e \mathbf{X}$ the dipole operator. $\Gamma$ consists of generalized damping terms, and we note that

$$
\Gamma_{i \alpha \alpha i} \equiv \Gamma_{i \alpha}=\frac{1}{2} A_{i \alpha}
$$

where $A_{i \alpha}$ is the Einstein coefficient for the transition from level $i$ to level $\alpha$. Thus, on a coarse-grained time scale, one obtains

$$
\begin{aligned}
\left|\psi_{A}(t)\right\rangle & =\mathcal{T} \exp \left\{-i \int_{0}^{t} d t^{\prime} H_{\text {cond }}\left(t^{\prime}\right) / \hbar\right\}\left|\psi_{A}(0)\right\rangle \\
& \equiv U_{\text {cond }}(t, 0)\left|\psi_{A}(0)\right\rangle .
\end{aligned}
$$

In an obvious extension to density matrices,

$$
\rho_{A}^{0}(t) \equiv U_{\text {cond }}(t, 0) \rho_{A}(0) U_{\text {cond }}(t, 0)^{\dagger}
$$

describes the sub-ensemble with no photon detection until time $t$, with the corresponding no-photon probability given by

$$
P_{0}(t)=\operatorname{tr} \rho_{A}^{0}(t) .
$$

Connection with the quantum Zeno effect: If one lets $\Delta t \rightarrow 0$ in Eq. (3), with $t=n \Delta t$ kept fixed, then one easily sees by the same calculation that the probability to find no photon until time $t$ goes to 1 and that one always stays in the no-photon subspace. This means that for $\Delta t \rightarrow 0$ the dynamics is frozen to the atomic subspace, and this is a particular form of the quantum Zeno effect.

Random path in Hilbert space for a single fluorescent atom: We can now distinguish different steps in the temporal behavior of our single atom under the above gedanken measurements.

(i) Until the detection of the first photon, our atom belongs to the sub-ensembles $\mathcal{E}_{0}^{(n \Delta t)}$ and hence is described by the (non-normalized) vector

$$
\left|\psi_{A}(t)\right\rangle=U_{\text {cond }}(t, 0)\left|\psi_{A}(0)\right\rangle .
$$

(ii) The first photon is detected at some (random) time $t_{1}$, according to the probability density

$$
w_{1}(t)=-\frac{d P_{0}(t)}{d t}=-\frac{d}{d t} \|\left|\psi_{A}(t)\right\rangle \|^{2}
$$


(iii) Jump: With the detection of a photon the atom has to be reset to the appropriate state. For example, a two-level atom will be in its ground state right after a photon detection. The general reset state for systems at rest has been determined in Refs. $[6,12]$ and is given in the next section. It may depend on $\left|\psi_{A}\left(t_{1}\right)\right\rangle$ where $t_{1}$ is the detection time of the photon.

(iv) From this reset state the time development then continues with $U_{\text {cond }}\left(t, t_{1}\right)$, until the detection of the next photon at the (random) time $t_{2}$. Then one has to reset (jump), and so on.

In this way one obtains a stochastic path in the Hilbert space of the atom. The stochasticity of this path is governed by quantum theory, and the path is called a quantum trajectory. The stochastic process underlying these trajectories is a jump process with values in a Hilbert space. If the reset state is always the same, e.g. the ground state, one has a renewal process. If the reset state depends on $\left|\psi_{A}\left(t_{i}\right)\right\rangle$ one has a Markov process only.

In case of a renewal process the parts of a trajectory between jumps behave like an ensemble created by repetition from a single system at stochastic times. In the general case the reset states can all be different so that this repetitive property is no longer true.

\section{Reset operation and Bloch equations}

Since a photon detection is a measurement this induces a state change (jump) and the atomic state or, more generally, the atomic density matrix $\rho(t)$ is reset to a new density matrix, denoted by $\mathcal{R}(\rho)$, up to normalization, where $\mathcal{R}$ is a superoperator acting on density matrices. Its general form is $[6]$

$$
\mathcal{R}(\rho)=\sum_{\substack{i j \ell m \\ i>j, \ell>m}}\left\{\Gamma_{j i \ell m}+\Gamma_{\ell m j i}\right\}|j\rangle\langle i|\rho| \ell\rangle\langle m|
$$

The normalization is such that the trace of $\mathcal{R}(\rho(t)) d t$ is the probability to find a photon in the time interval $d t$. For a two-level system one easily finds $\mathcal{R}(\rho) \propto|1\rangle\langle 1|$, i.e. the intuitively expected ground state. In this case the result of the resetting is a pure state, which simplifies numerical simulations.

As shown in Ref. [6] the ensemble of all possible random paths (quantum trajectories) leads to a reduced density matrix for the ensemble of atoms which satisfies the usual optical Bloch equations. This is a nice consistency check [19] and can be seen as folllows.

We consider a large ensemble $\left(N_{0} \rightarrow \infty\right)$ of quantum trajectories. They will have jumps at different times. Consider time $t$, and let the ensemble of systems (atoms) at $t$ be described by the density matrix $\rho(t)$. Then in $(t, t+d t)$ (coarsegrained timescale!) a subensemble of systems will have no photon detection, and this subensemble is described, including relative size, by

$$
U_{\text {cond }}(t, t+d t) \rho(t) U_{\text {cond }}^{+}(t, t+d t)
$$


and those with a photon detection are described by (including relative size)

$$
\mathcal{R}(\rho(t)) d t
$$

Hence one has

$$
\rho(t+d t)=\rho(t)-\frac{i}{\hbar}\left\{H_{\text {cond }} \rho-\rho H_{\text {cond }}^{+}\right\} d t+\mathcal{R}(\rho) d t
$$

From this one immediately obtains

$$
\dot{\rho}=-\frac{i}{\hbar}\left\{H_{\text {cond }} \rho-\rho H_{\text {cond }}^{+}\right\}+\mathcal{R}(\rho)
$$

This is a compact form of the Bloch equations for the general $N$-level system, and the usual two-level case is easily deduced from this.

\section{Remarks}

1. If the Bloch equations are given, and either $H_{\text {cond }}$ or $\mathcal{R}$, one can read off the other from (19). There are efficient ways to derive master (Bloch) equations (e. g. the projector method) and then one can guess $H_{\text {cond }}$ and $\mathcal{R}$ from it.

2. The quantum jump approach lends itself to simulations of trajectories, and by averaging many of those one can obtain solutions of the Bloch equations. If $\mathcal{R}$ resets to pure states one has to work in dimensions $N$ of the atomic Hilbert space instead of dimensions $N^{2}$ as for Bloch equations. For large $N$ tremendous numerical advantages arise. The jump operation $\mathcal{R}$ will in general give a density matrix, but one can usually write it as

$$
\mathcal{R}(\rho)=\sum_{i} R_{i} \rho R_{i}^{+}
$$

where $R_{i}$ are operators. One can then simulate a pure state $R_{i}|\psi(t)\rangle$ in each jump. The resulting trajectories of pure states give the same Bloch equations.

\section{Systems with quantized center-of-mass motion}

As an example we consider a two-level system with quantized $\mathrm{cm}$ motion, interacting with a classical laser and the quantized radiation field. The corresponding Hamiltonian is of the form

$$
H=\hat{\mathbf{P}} / 2 m+\hbar \omega_{21}|2\rangle\langle 2|+\sum \hbar \omega_{k} \hat{a}_{\mathbf{k} \lambda}+\hat{a}_{\mathbf{k} \lambda}+\hat{\mathbf{D}} \cdot \hat{\mathbf{E}}(\hat{\mathbf{X}}, 0)+\hat{\mathbf{D}} \cdot \mathbf{E}_{L}(\hat{\mathbf{X}}, t)
$$

where ^ denotes an operator. The classical laser field is of the form

$$
\mathbf{E}_{L}(\mathbf{x}, t)=\operatorname{Re} \mathbf{E}_{0}(\mathbf{x}) e^{-i \omega_{L} t}
$$


where we allow for a position-dependent laser amplitude. With

$$
g_{k \lambda}=i e \sqrt{\frac{\omega_{k}}{2 \varepsilon_{o} \hbar V}} \varepsilon_{\mathbf{k} \lambda} \cdot \mathbf{D}_{12}
$$

one then has in the laser-adapted interaction picture and in the rotating-wave approximation (RWA),

$$
\begin{aligned}
H_{I}^{L}= & -\hbar \Delta|2\rangle\langle 2| \\
& +|1\rangle\langle 2| \sum g_{\mathbf{k} \lambda} \hat{a}_{k, \lambda} \exp \left\{-i\left(\omega_{k}-\omega_{L}\right) t\right\} \exp \{i \mathbf{k} \cdot(\hat{\mathbf{X}}+\hat{\mathbf{P}} t / m)\}+\text { h.c. } \\
& +\frac{1}{2}|1\rangle\langle 2| \Omega(\hat{\mathbf{X}}+\hat{\mathbf{P}} t / m)+\text { h.c. }
\end{aligned}
$$

where $\Delta=\omega_{L}-\omega_{21}$ and the position-dependent Rabi frequency is

$$
\Omega(\mathbf{x})=\langle 1|\hat{\mathbf{D}}| 2\rangle \cdot \mathbf{E}_{0}(\mathbf{x}) .
$$

We consider the Dyson series of the time-development operator from $t$ to $t+\Delta t$ and keep only terms proportional to $\Delta t$. Then the laser contributes to the first order only. and the second-order term in the time-development operator becomes

$$
\begin{aligned}
\left(-\frac{i}{\hbar}\right)^{2}\left\langle 0_{p h}\right| \int_{t}^{t+\Delta t} d t^{\prime} & \int_{t}^{t^{\prime}} d t^{\prime \prime} H_{I}^{L}\left(t^{\prime}\right) H_{I}^{L}\left(t^{\prime \prime}\right)\left|0_{p h}\right\rangle= \\
\frac{1}{\hbar^{2}}|2\rangle\langle 2| & \int_{t}^{t+\Delta t} d t^{\prime} \int_{t}^{t^{\prime}} d t^{\prime \prime} \sum_{\mathbf{k} \lambda}\left|g_{\mathbf{k} \lambda}\right|^{2} \exp \left\{i\left(\omega_{k}-\omega_{L}\right)(t-t)\right\} \\
& \exp \left\{i \mathbf{k} \cdot\left(\hat{\mathbf{X}}+\hat{\mathbf{P}} t^{\prime} / m\right)\right\} \exp \left\{-i \mathbf{k} \cdot\left(\hat{\mathbf{X}}+\hat{\mathbf{P}} t^{\prime \prime} / m\right)\right\}
\end{aligned}
$$

where the laser terms have been omitted since they are proportional to $(\Delta t)^{2}$. We note that

$$
\left.\hat{\mathbf{X}}\left(t^{\prime}\right)=\hat{\mathbf{X}}+\hat{\mathbf{P}} t^{\prime} / m\right)
$$

is the time development of $\hat{\mathbf{X}}$ in the free Heisenberg picture of the $\mathrm{cm}$ motion of the system. If $\Delta t$ is small and the atom not moving too fast, then

$$
\hat{\mathbf{X}}\left(t^{\prime}\right) \approx \hat{\mathbf{X}}\left(t^{\prime \prime}\right)
$$

and the last two exponentials cancel. This leads to the damping term $-\frac{1}{2} A|2\rangle\langle 2|$ as in the two-level case without $\mathrm{cm}$ motion and one obtains as conditional Hamiltonian

$$
H_{\text {cond }}^{I}=\hat{\mathbf{P}}^{2} / 2 m-\hbar \Delta|2\rangle\left\langle 2\left|-\frac{i}{2} \hbar A\right| 2\right\rangle\left\langle 2\left|+\frac{\hbar}{2} \Omega(\hat{\mathbf{X}})\right| 1\right\rangle\langle 2|+\text { h.c. }
$$

The reset or jump operation looks somewhat different now. The atomic density operator $\rho$ now describes both the $\mathrm{cm}$ motion and the internal two-level degrees of freedom. Thus, taking matrix elements with momentum eigenvectors of the $\mathrm{cm}$ motion,

$$
\rho\left(\mathbf{p}, \mathbf{p}^{\prime}\right) \equiv\left\langle\mathbf{p}|\rho| \mathbf{p}^{\prime}\right\rangle
$$


one obtains an operator for the internal degrees of freedom, i.e. a $2 \times 2$ matrix. With the Hamiltonian in Eq. (21) a calculation similar to that in Ref. [6] gives for the reset operation after a photon detection

$$
\left\langle\mathbf{p}|\mathcal{R}(\rho)| \mathbf{p}^{\prime}\right\rangle=A|1\rangle\langle 1| \int d \Omega_{\mathbf{k}}\left(1-\frac{|\hat{\mathbf{k}} \cdot \mathbf{D}|^{2}}{\mathbf{D}^{2}}\right)\left\langle 2\left|\rho\left(\mathbf{p}+\hbar \omega_{21} \hat{\mathbf{k}} / c, \mathbf{p}^{\prime}+\hbar \omega_{21} \hat{\mathbf{k}} / c\right)\right| 2\right\rangle
$$

where the integration is over the unit vectors $\hat{\mathbf{k}}$. The first factor in the integral is the usual dipole emission characteristics and the terms $\hbar \omega_{21} \hat{\mathbf{k}} / c$ yield momentum conservation after the photon emission. We note that the resulting reset matrix is a pure state only for internal degrees of freedom but not for the $\mathrm{cm}$ variables, even if the density matrix before the photon detection is a pure state.

Instead of asking for the detection of any photon one may ask a photon detection in a given direction $\hat{\mathbf{k}}$. Then the reset operation is given by

$$
\left\langle\mathbf{p}\left|\mathcal{R}_{\hat{\mathbf{k}}}(\rho)\right| \mathbf{p}^{\prime}\right\rangle=A|1\rangle\langle 1|\left(1-\frac{|\hat{\mathbf{k}} \cdot \mathbf{D}|^{2}}{\mathbf{D}^{2}}\right)\left\langle 2\left|\rho\left(\mathbf{p}+\hbar \omega_{21} \hat{\mathbf{k}} / c, \mathbf{p}^{\prime}+\hbar \omega_{21} \hat{\mathbf{k}} / c\right)\right| 2\right\rangle
$$

and the probability to detect a photon with direction in the solid angle $d \Omega_{\mathbf{k}}$ in the time interval $d t$ is given by

$$
\operatorname{Tr} \mathcal{R}_{\hat{\mathbf{k}}}(\rho) d \Omega_{\mathbf{k}} d t
$$

If $\rho$ is a pure state in momentum space this is again a pure state. Averaging Eq. (27) over all directions gives the reset matrix in Eq. (26).

\section{Application to quantum arrival times}

An important open problem in quantum theory is the question of how to formulate the notion of "arrival time" of a particle, such as an atom, at a given location, i.e. the time instant of its first detection there. This is clearly a very physical question, but when the extension and spreading of the wave packet is taken into account, a satisfactory formulation is far from obvious. The problem of time in quantum mechanics, both for time instants and time durations such as dwell time, has received a great deal of theoretical attention recently [20, 21]. When the translational motion of the particle can be treated classically, a full quantum analysis of arrival time is in fact not necessary. This is the case for fast particles, and therefore arrival times are presently measured mostly by means of time-of-flight techniques, whose analysis is carried out in terms of classical mechanics. Problems, though, arise for slow particles for which the finite extent of the wavefunction and its spreading become relevant, such as for cooled atoms dropping out of a trap. Then a quantum theoretical point of view is needed. It is therefore important to find out when the classical approximations fail and to propose measurement procedures for arrival times in the quantum case. Since the theoretical definition of a quantum arrival time is still subject to debate it is necessary to determine what exactly such measurement procedures are measuring and to compare such operational approaches with the existing, more abstract and axiomatic, theories. 
An experimentally very natural approach to determine the arrival time of an atom is by quantum optical means. A region of space may be illuminated by a laser and upon entering the region an atom will start emitting photons. The first photon emission can be taken as a measure of the arrival time of the atom in that region.

It is easiest to first study the one-dimensional case and use the corresponding equations of the preceding section. Eq. (25) then simply becomes one-dimensional in $\mathrm{p}$ and $\mathrm{x}$. Illuminating the half axis $x>0$ perpendicularly by a laser, the Rabi frequency $\Omega(x)$ becomes a multiple of the step function $\Theta(x)$. In Ref. [22] the corresponding conditional time development has been solved explicitly and the distribution of the first-photon times have been calculated. If one deducts by a deconvolution technique the delays due to finite pumping time one obtains in the limit of weak pumping a surprising result for the arrival time distribution, namely the usual well known quantum mechanical probablity flux.

\section{References}

[1] H.G. Dehmelt, Bull. Am. Phys. Soc. 20, 60

[2] J. C. Bergquist, R. G. Hulet, W. M. Itano and D. J. Wineland, Phys. Rev. Lett. 57, 1699 (1986); Th. Sauter, R. Blatt, W. Neuhauser, and P.E. Toschek, Opt. Comm. 60, 287 (1986); W. Nagourney, J. Sandberg, and H. Dehmelt, Phys. Rev. Lett. 56, 2797 (1986); J.C. Bergquist, W.M. Itano, R.G. Hulet, and D.J. Wineland, Phys. Script. T22, 79 (1988)

[3] R.J. Cook and H.J. Kimble, Phys. Rev. Lett. 54, 1023 (1985); C. Cohen-Tannoudji and J. Dalibard, Europhysics Lett. 1, 441 (1986); A. Schenzle, R.G. DeVoe, and R.G. Brewer, Phys. Rev. A 33, 2127 (1986); J. Javanainen, Phys. Rev. A 33, 2121 (1986); D.T. Pegg, R. Loudon, and P.L. Knight, Phys. Rev. A 33, 4085 (1986), G. Nienhuis, Phys. Rev. A 35, 4639 (1987); P. Zoller, M. Marte, and D.J. Walls, Phys. Rev. A 35, 198 (1987); S. Reynaud, J. Dalibard, and C. Cohen-Tannoudji, IEEE Journal of Quantum Electronics 24, 1395 (1988);M. Porrati and S. Putterman, Phys. Rev. A 36, 925 (1987) and A 39, 3010 (1989); M.S. Kim and P.L. Knight, Phys. Rev. A 40, $215(1989)(1975)$

[4] G.C. Hegerfeldt and T.S. Wilser, in: Classical and Quantum Systems. Proceedings of the II. International Wigner Symposium, July 1991, edited by H.D. Doebner, W.Scherer, and F. Schroeck; World Scientific (Singapore 1992), p. 104.

[5] T.S. Wilser, Doctoral Dissertation, University of Göttingen (1991).

[6] G.C. Hegerfeldt, Phys. Rev. A 47, 449 (1993).

[7] J. Dalibard, Y. Castin, and K. Mølmer, Phys. Lett. 68, 580 (1992)

[8] H. Carmichael, An Open Systems Approach to Quantum Optics, Lecture Notes in Physics, Springer (Berlin 1993)

[9] G.C. Hegerfeldt and M.B. Plenio, Phys. Rev. A 45, 373 (1992)

[10] G.C. Hegerfeldt and M.B. Plenio, Phys. Rev. A 47, 2186 (1993)

[11] G.C. Hegerfeldt and M.B. Plenio, Quantum Optics 6, 15 (1994) 
[12] G.C. Hegerfeldt and D.G. Sondermann, Quantum Semiclass. Opt. 8, 121 (1996)

[13] M.B. Plenio and P.L. Knight, Rev. Mod. Phys. 70, 101 (1998)

[14] G.C. Hegerfeldt, Fortschr. Phys. 46, 596 (1998)

[15] Alternatively one can use the Markov assumption. Cf., e.g., R. Reibold, J. Phys. A 26, 179 (1993). See also M. Porrati and S. Putterman, Phys. Rev. A 39, 3010 (1989).

[16] As will be pointed out further below, the calculation of the photon emission probabilities will automatically give zero for the present case when $\Delta t \rightarrow 0$. This is a special manifestation of the quantum Zeno effect. Extensive references on this effect can be found in A. Beige and G.C. Hegerfeldt, Phys. Rev. A 53 (1996) and in A. Beige, G.C. Hegerfeldt, and D.G. Sondermann, Found. Phys. 27, 1671 (1997).

[17] For a similar requirement cf. P.A.M. Dirac, The Principles of Quantum Mechanics, 4th Ed., Clarendon Press (Oxford 1959), p. 181.

[18] The projection postulate as commonly used nowadays is due to G. Lüders, Ann. Phys. 8, 323 (1951). For observables with degenerate eigenvalues his formulation differs from that of J. von Neumann, Mathematische Grundlagen der Quantenmechanik, Springer (Berlin 1932) (English translation: Mathematical Foundations of Quantum Mechanics, Princeton University Press, 1955), Chapter V.1. The projection postulate intends to describe the effects of an ideal measurement on the state of a system, and it has been widely regarded as a useful tool. Cf. also the remark in P.A.M. Dirac, The Principles of Quantum Mechanics, 1st Ed., Clarendon Press (Oxford 1930), p. 49 , about measurements causing minimal disturbance. In later editions this passage has been omitted.

[19] The quantum trajectories can therefore be used to obtain numerical solutions of the Bloch equations by simulations. This was stressed in Ref. [7]. For the simulation of the Bloch equations also other trajectories can be used which do not have the same physical meaning as in the above approach. Cf. e.g. N. Gisin, Phys. Rev. Lett.52, 1657 (1984); N. Gisin and I.C. Percival, J. Phys. A: Math. Gen. 25, 5677 (1992) and the survey by D.G. Sondermann, in: Nonlinear, deformed and irreversible quantum systems, edited by H.-D. Doebner, V.K. Dobrev, and P. Nattermann, World Scientific 1995; p. 273.

[20] J. G. Muga and C. R. Leavens, Phys. Rep. 338, 353 (2000).

[21] J. G. Muga, R. Sala and I. L. Egusquiza (eds.), Time in Quantum Mechanics (Springer, Berlin, 2002).

[22] J. A. Damborenea, I. L. Egusquiza, G. C. Hegerfeldt, and J. G. Muga, Phys. Rev. A 66, $052104(2002)$ 